Jesusa M. Santos, MD

Department of Otorhinolaryngology

Head and Neck Surgery

Jose R. Reyes Memorial Medical Center
Correspondence: Dr. Jesusa M. Santos Department of Otorhinolaryngology Head and Neck Surgery

Jose R. Reyes Memorial Medical Center

San Lazaro Compound, Rizal Avenue

Sta. Cruz, Manila 1003

Philippines

Phone: (632) 87119491 local 320

E-mail: zette_jms@yahoo.com

The author declared that this represents original materia that is not being considered for publication or has not been published or accepted for publication elsewhere, in full or in part, in print or electronic media; that the manuscript has been read and approved by the author, that the requirements for authorship have been met by the author, and that the author believes that the manuscript represents honest work.

Disclosures: The author signed disclosures that there are no financial or other (including personal) relationships, intellectual passion, political or religious beliefs, and institutional affiliations that might lead to a conflict of interest.

Presented at the Philippine Society of Otolaryngology Head and Neck Surgery Descriptive Research Contest. December 5, 2019. Palawan Ballroom, Edsa Shangri La Hotel, Mandaluyong City.

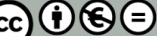

\title{
Prevalence of Diabetes Mellitus and Clinicodemographic Profiles of Patients with Head and Neck Infections in a Philippine Tertiary Government Hospital
}

\begin{abstract}
Objective: To determine the prevalence of diabetes mellitus among patients admitted for head and neck infections and describe their clinicodemographic features.
\end{abstract}

\section{Methods:}

$\begin{array}{ll}\text { Design: } & \text { Retrospective Case Series } \\ \text { Setting: } & \text { Tertiary Government Training Hospital } \\ \text { Participants: } & \text { Forty-two (42) patients }\end{array}$

Results: Out of 211 adult patients admitted for head and neck infections during the study period, 42 (20\%) were diagnosed to have concomitant diabetes mellitus (DM). Only $6(14 \%)$ were known to have DM before admission while $28(67 \%)$ were found to have DM only after admission. The most common site of infection was the neck $(11 ; 26 \%)$. More than half of the patients (24; 57.1\%) had infections in the head only, 17 (40.5\%) had infections in the neck only, and 1 $(2.4 \%)$ had infections in both the head and neck regions. Among these, 26 (61.9\%) had infection in one site only, 15 (35.7\%) had infections in two sites and one (2.4\%) had infections in three sites. The majority $(28 ; 66.7 \%)$ had an unknown etiology of infection with spontaneous appearance of redness and swelling in the involved area. Six (14.3\%) were odontogenic, five (11.9\%) were due to skin trauma, and three $(7.1 \%)$ were due to nasal mucosal trauma.

Available cultures in 14 patients revealed 12 (86\%) with aerobic microorganisms and two (14\%) with anaerobic growths. Half of the aerobic cultures grew K. pneumoniae. All patients were started on empiric intravenous antibiotics and over half of them (52.4\%) needed surgical management. More than half $(27 ; 64.3 \%)$ suffered from diabetic head and neck-associated complications, categorized into orbital (56\%), organ/systemic (26\%), local (11\%), and neural (7\%). Despite these complications, the majority $(37,86 \%)$ went home improved while five (12\%) expired.

Conclusion: This study found that $20 \%$ of patients admitted for head and neck infections during the study period had concurrent DM. Guided by known clinicodemographic characteristics, patients admitted with suspicious head and neck infections must be promptly screened for concomitant DM and properly managed before substantial morbidity and death ensue. Otolaryngologists - head and neck surgeons, endocrinologists, general practitioners and patients alike must be cognizant of diabetic head and neck infections so that they can be prevented or managed early before complications set in.

Keywords: diabetes mellitus; head and neck infections; diabetic infections 


\section{ORIGINAL ARTICLES}

Over the years, lifestyle-related/non-communicable diseases such as DM have risen to be among the top causes of morbidity and mortality globally and locally. 'In the Philippines, there were $3,721,900$ cases of diabetes in 2017 with a $6.2 \%$ prevalence in the adult population. ${ }^{2}$ Diabetic-related complications include heart attack, stroke, kidney failure, foot and leg gangrene, vision loss, nerve damage and infections all of which bring about substantial financial burden and extended hospital stays. ${ }^{3}$

Diabetes increases susceptibility to various infections that can affect any region of the body including the head and neck. Head and neck infections include malignant otitis externa, deep neck space abscesses, parotid abscesses, rhino-cerebral mucormycosis, and others. ${ }^{4}$ These bring potential serious complications like hearing loss, airway obstruction, facial palsy and even blindness. Diabetics are predisposed to greater infection risk primarily due to hyperglycemia and acidemia which exacerbate impairments in humoral immunity and polymorphonuclear leukocyte and lymphocyte functions. ${ }^{5}$ These may precipitate metabolic derangements, and conversely, the metabolic derangements of diabetes may facilitate infection. ${ }^{6}$ The treatment of diabetes - associated head and neck infections is quite challenging and involves prolonged anti-microbial therapy, glycemic control and even major surgical debridement.

To the best of my knowledge based on a search of HERDIN, MEDLINE (PubMed) and Google Scholar using the search terms "diabetes," "infections," "head and neck," "diabetic head and neck infections," and "Philippines" there are no previous studies on the prevalence of diabetes among patients admitted for head and neck infections in the Philippines. Thus, this study aims to determine the prevalence of diabetes among patients admitted for head and neck infections in our ward over a 5-year period, and to describe their clinicodemographic characteristics.

\section{METHODS}

After obtaining approval of the Jose R. Reyes Memorial Medical Center Institutional Review Board (IRB Protocol No. 2019-085), this 5 -year retrospective series was conducted by reviewing the available ward census of the Department of Otorhinolaryngology - Head and Neck Surgery for the period between January 2014 to December 2018.

The source population were records of all patients admitted for head and neck infections at the Department of Otorhinolaryngology - Head and Neck Surgery. Of these, records of adult patients with DM admitted for head and neck infections were considered for inclusion if they met any of the following criteria: glycosylated hemoglobin test (HbA1c) level of $6.5 \%$ and higher, or fasting blood sugar of $126 \mathrm{mg} / \mathrm{dl}$ $(7.0 \mathrm{mmol} / \mathrm{L})$ or above, or random glucose test of $200 \mathrm{mg} / \mathrm{dl}$ or higher, or were listed as a known diabetic with maintenance medications. Incomplete records were excluded.

The clinicodemographic profile of included patients such as age, gender, site of infection, multiplicity of sites of infection, etiology of infection, culture studies (if available), blood glucose level, treatment modality (antibiotics, other medications, surgery), length of hospital stay, complications and outcomes were extracted from the records.

Data was recorded and tabulated using Microsoft Excel ${ }^{\circ}$ for Mac Version 16.4 (Microsoft Corp., Redmond WA, USA), while STATA version 14 (StataCorp LLC, College Station TX, USA) was used in data analysis. Descriptive statistics were employed to determine the mean \pm standard deviation for continuous variables and the proportions for categorical variables.

\section{RESULTS}

A total of 211 adult patients were admitted for head and neck infections during the study period and 42 (20\%) of these patients were identified to have concomitant DM. There were 24 (57\%) males and 18 (43\%) females, with ages ranging from 25 to 76 years old (mean age 48 years old). There was bimodal distribution with $16(38 \%)$ aged 51 to 60 years old and $11(26 \%)$ aged 31 to 40 years old. Eight (19\%) were aged between 41-50, four (10\%) between 61-70, two (5\%) between 21-30, and one (2\%) between 71-80 years of age.

Only six patients (14\%) were known to have DM before admission. Following admission, 28 patients (67\%) were identified to have DM based on elevated $\mathrm{HbA} 1 \mathrm{c}$ levels, six (14\%) based on elevated FBS level while two (5\%) had elevated random blood sugar. The mean $\mathrm{HbA1c}$ level was $10.7 \%$, with the highest level at $18 \%$ and lowest at $6.6 \%$.

The most common site of infection was the neck $(11 ; 26 \%)$ followed by the nose $(9 ; 21 \%)$, ear $(4 ; 9.5 \%)$, submandibular area $(3 ; 7 \%)$, submental area, upper lip, buccal and retropharyngeal areas ( 2 each; $5 \%$ each), and temporal area, epiglottis, tonsil, parotid, frontal area, cheek, and lower lip (1 each; 2\% each). Among the patients, 26 (61.9\%) had infection in one site only, while $15(35.7 \%)$ had infections in two sites. Only one (2.4\%) had infections in three sites. Infections in the head region only were observed in more than half of the patients (24; $57.1 \%)$, while infections in the neck region only were observed in 17 $(40.5 \%)$. Only one patient (2.4\%) had infections in both the head and neck regions.

A majority $(28 ; 66.7 \%)$ of the patients had an unknown etiology of infection with spontaneous appearance of redness and swelling in the involved area. Six (14.3\%) were odontogenic (associated with dental caries) and $5(11.9 \%)$ were due to skin trauma. Three $(7.1 \%)$ were due to nasal mucosal trauma such as plucking of nasal vibrissae. (Figure 1) 


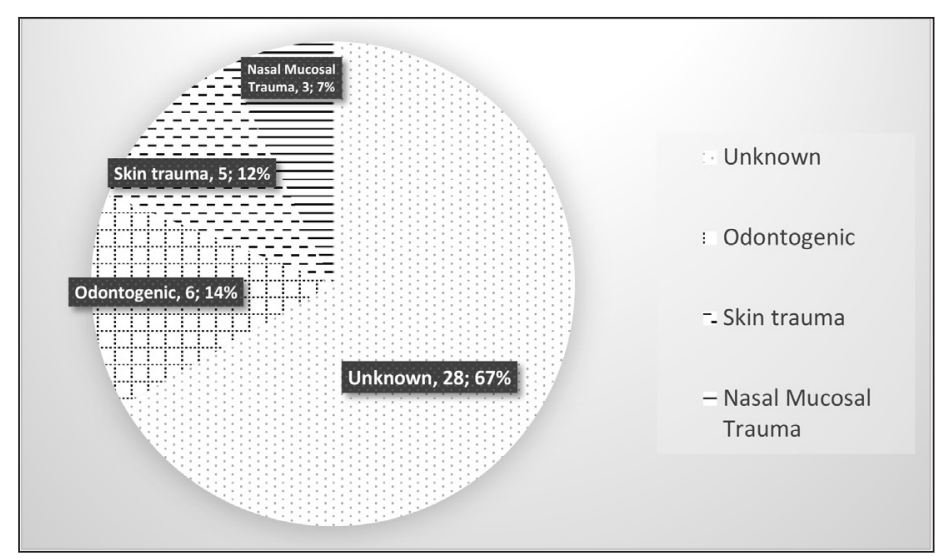

Figure 1. Etiologies of Infection. Most are from unknown causes (dotted), followed by odontogenic (grid), skin trauma (dashed) and nasal mucosal trauma (solid lines).

Culture results were available in 14 patients (collected from surgery or needle aspiration). Twelve (86\%) had growths of aerobic microorganisms while two (14\%) had anaerobic microbial growths. Among the aerobic cultures, six grew K. pneumoniae, three grew S. aureus and one each grew Salmonella, Staphylococcus epidermidis and Pseudomonas. Among the anaerobes there were one sample each of Arizona sp. and Enterobacter aerogenes.

All of the patients were started on empiric intravenous antibiotics until culture studies led to definitive treatment. Antibiotics and number of patients were: Oxacillin + Gentamicin (10; 24\%), Piperacillin Tazobactam (8; 19\%), Oxacillin (4; 10\%), Penicillin G + Metronidazole $\quad(3 ; 7 \%)$, Piperacillin Tazobactam + Clindamycin (3; 7\%), Ampicillin Sulbactam $(2 ; 5 \%)$ and one each (2\% each), Sultamicillin + Metronidazole, Penicillin G, Ceftriaxone + Metronidazole, Oxacillin + Ciprofloxacin, Piperacillin Tazobactam + Metronidazole, Piperacillin Tazobactam + Meropenem, Ceftriaxone, Vancomycin + Ceftazidime, Ciprofloxacin, Clindamycin, Clindamycin + Ceftazidime, Ceftriaxone + Oxacillin + Metronidazole. More than half of the patients (27; 64.2\%) were on insulin therapy. Ten (23.8\%) were taking oral hypoglycemic agents and five (12\%) were taking combination therapy (insulin SQ/IV + oral hypoglycemic agents).

Of the 42 patients, half $(22 ; 52.4 \%)$ required surgical intervention. Of these 22, 14 (63\%) underwent incision and drainage under local anesthesia while four patients (18\%) with deeper abscesses underwent incision and drainage under general anesthesia. One patient underwent direct laryngoscopy for mapping of the extent and drainage of the retropharyngeal abscess. Three patients (14\%) underwent wound debridement under general anesthesia due to substantial amounts of necrotic tissues in the affected site.

More than half $(27 ; 64.3 \%)$ of the patients had complications associated with diabetic head and neck infections. Of these, 15 (56\%) had orbital complications. These were pre-septal cellulitis (12), cavernous sinus thrombosis (2), and orbital cellulitis (1). Seven (26\%) had organ/systemic complications: mediastinitis (1), pleural effusion (1), hypoxemic encephalopathy (1) and sepsis (1), with three having concomitant impending upper airway obstruction. Three (11\%) had local complications such as skin defect (2) and saddle nose deformity (1). Two (7\%) had neural complications, facial palsy (1) and hearing loss (1). Patients who had pre-septal cellulitis initially had infections of the nose, upper lip, temporal area, buccal and frontal area, respectively. The patient with saddle nose deformity had a nasal septal abscess (and subsequently underwent septorhinoplasty). The two patients who had lateral neck skin defects were allowed to heal by secondary intention. The patient with hypoxemic encephalopathy developed from airway obstruction due to the significant retropharyngeal abscess. The patient with hearing loss had malignant otitis externa that led to external auditory canal stenosis.

Despite complications, 36 patients (86\%) went home improved, one (2\%) was readmitted due to recurrence of lateral deep neck abscess, and five patients (12\%) expired. The mean duration of hospital stay for diabetic patients with head and neck infections was 14 days, with the longest hospital stay at 32 days and the shortest at less than one day.

\section{DISCUSSION}

The prevalence of DM among patients with head and neck infections in our institution during the 5 -year study period was $20 \%$. This suggests that one out of five patients admitted for head and neck infections in the hospital has diabetes, although most might only be diagnosed to have DM following admission. Only a small proportion of the patients in this present series knew that they had diabetics on admission.

Patients admitted for head and neck infections must be properly screened for concomitant DM to facilitate appropriate management. Smit's study reported that diabetic patients with poor management of their blood glucose level((HbA1c > 7\%) show a greater risk for infections which are relatively more serious. ${ }^{7}$ Hyperglycemia has been reported to impair the immune functions of hosts by increasing the virulence of some pathogens, lowering the production of interleukins, immobilization of leukocytes and reduced chemotaxis and phagocytic activity. ${ }^{8}$ These render the patients at higher risk for severe or invasive infections involving any organ or regions of the body but are also common in the head and neck region.?

In this study, the majority of the patients were in their fifth decade. This can be attributed to the older age group being more predisposed to systemic illness such as DM and a decline in their immune function. ${ }^{9}$ This is also reflective of the age group 50-59 identified in the Global Report on Diabetes by WHO. ${ }^{3}$ Synergistically, poorly controlled diabetes and aging subject a person to increased infection risk. 


\section{ORIGINAL ARTICLES}

The most commonly involved site of infection identified in this study was the neck but any part of the head and neck region such as the ear, nose, face, lip, and throat can be primary sites of infection as well. These sites are infrequently identified as potential nidus for diabetic-related complications, with blindness, kidney failure, cardiovascular events and lower limb amputation as the most commonly identified consequences that impact significantly on diabetic patients. ${ }^{3}$ Several studies revealed that diabetes mellitus is the most commonly associated systemic disease in deep neck infections with a unique clinical picture in comparison with those without diabetes mellitus. ${ }^{10,11}$

In this study, most of the patients were admitted due to unknown etiology of infection followed by odontogenic infections. El-Sayed et al. stated that the causes of infections remain obscure in diabetic patients because of possible resolution of the infectious foci or because subclinical infectious foci exist. ${ }^{12} \mathrm{~A}$ study by Vieira and Stocks theorized that poor oral hygiene and low socio-economic status probably accounted for the odontogenic causes. ${ }^{13}$ Caqueiro et al. stated that the hyperglycemic state of patients seems to be central in the initiation of the pathophysiology of diabetic head and neck infections. ${ }^{8}$

Klebsiella pneumoniae was the most common aerobic bacteria isolated from purulent exudates of our patients followed by Staphylococcus aureus while anaerobic microbial growth revealed Arizona sp. and Enterobacter aerogenes. This shows that diabetic patients are prone to infections with various causative organisms. A German study revealed that $K$. pneumoniae in deep neck infection patients with diabetes is common due to increased oropharyngeal colonization by gram-negative bacilli and defects on host defenses, specifically the phagocytic function which predispose them to invasive type of infections. ${ }^{14}$ Culture and sensitivity is a great tool in giving appropriate antimicrobial therapy in these types of serious bacterial infections. In vitro data show that a wide-range of beta-lactams, aminoglycosides and quinolones are useful treatment for Klebsiella infections. 15,16,17

Because only six (14\%) of the patients were known to have DM on admission, screening for the blood glucose level was requested from patients who presented with unconfirmed and more severe forms of infection of the head and neck. The mean $\mathrm{HbA1c}$ level of the diabetic patients with this specific test was $10.7 \%$ with highest level at $18 \%$. Hyperglycemia causes protein glycation which can cause impairment of host proteins involved in complement activation, bacterial uptake, phagocytic killing, and scavenging of bio-limiting nutrients and change the binding of host surface receptors for pathogens..$^{18}$ Moreover, glycation of immunoglobulin occurs in patients with diabetes in proportion with the increase in $\mathrm{HbA} 1 \mathrm{c}$, and this may harm the biological function of the antibodies. ${ }^{19}$ Hence, confinement of the infection by the host's immune system is also ineffective leading to morbid complications, extensive infections and even death. ${ }^{6}$

Intravenous antibiotics were started empirically based on site of infection and organism that was most likely to be the causative agent while definitive antibiotics were given for patients with culture studies. Blood glucose lowering agents were also needed to optimize treatment. Almost half of the diabetic patients in our study suffering from head and neck infections required surgical treatment. Holkom et al. documented that diabetic patients with abscess formation with extended space infection underwent surgery more than non-diabetic patients, with the difference being statistically significant. ${ }^{20}$ This aggressive mode of treatment was expedited since conservative medical treatment failed to completely resolve the deep-seated infections. In developing countries like the Philippines, it is common for patients to seek medical consultation in the advanced stages of the clinical spectrum when complications have already set in.

The mean hospitalization period among our patients was 14 days. During their hospital stay glycemic control was aimed to maximize the effect of medical and surgical treatment. Daily wound care by the surgeon was also necessary since some needed regular debridement and the cases of deep neck infections were left to heal by secondary intention, necessitating prolonged hospital stay. A multitude of complications ranging from local, orbital, nerve and organ/systemic affectation were seen in our patients. Huang et al. concluded that diabetic patients tend to have more frequent complications from head and neck infections which may be related to high percentage of extended involvement rendering them less suitable for conservative treatment and prolonged hospital stay. ${ }^{21}$ Within the age range of 25 76 years old, five (12\%) of our study patients succumbed to death. This rate is higher than the worldwide statistics of $7-8 \%$ deaths due to high blood glucose among those aged 20-69 presented by the WHO. Hence, at the individual level, the WHO suggested that intensive interventions to improve diet and physical activity can prevent or delay the onset of diabetes in people at high risk. ${ }^{3}$ Due to disturbances of their immune system and hyperglycemic state, a high fatal outcome of infectious diseases in diabetic patients was reported in an Israeli study by Leibovici. ${ }^{22}$ Huang et al. also stated that risk of mortality related to infection in a diabetic adult patient is greater than that of a cardiovascular disease patient. ${ }^{17}$ However, when managed appropriately, maximal medical and surgical treatment and glycemic control can promote full recovery of patients. ${ }^{17}$

The major limitation of this study is the small number of patients gathered during the study period. The source population itself was biased as it consisted of admissions for head and neck infections. 
A future multi-center prospective study can be conducted to increase the study population. The source population could be expanded to include all patients with DM and identify those with head and neck infections in particular (rather than starting with all patients with head and neck infections and identifying those with underlying DM). The glycosylated hemoglobin ( $\mathrm{HbA} 1 \mathrm{c}$ ) level was also not available in all patients (particularly those who expired within less than a day of admission). Association studies could have been performed if all patients had the same DM indicators such as their $\mathrm{HbA1c}$ level. An awareness program is needed to increase the knowledge of lay persons, general practitioners and otolaryngologists - head and neck surgeons about diabetic head and neck infections, their clinical picture and implications on patients, their families, physicians, hospitals and communities.

This study focused on DM as a prevalent disease seen among patients with head and neck infections. The general public and physicians should be made aware of the neglected presentation of beginning erythema or swelling on any part of the head and neck without prior source as a possible diabetic head and neck infection. Systemic antibiotics covering K. pneumoniae should be considered since majority of the infections grew these bacteria. Primary prevention is still best by observing strict dietary control, regular exercise and secondary prevention by encouraging patient with known DM to have good compliance with anti-diabetic medications and regular screening of the body giving importance to the head and neck region as well.

Diabetic head and neck infections can lead to significant disability and even premature death. The role of an otolaryngologist may be almost as essential as that of the endocrinologist in handling such cases. The results of the prevalence and clinicodemographic profile of diabetic patients admitted for head and neck infections in this study may be a basis for future studies that can establish the association of hyperglycemic state to the characteristics seen in the infections of the patients which can later on create an awareness program that can educate patients and medical practitioners alike on the alarming consequence of diabetes in general and diabetic head and neck infections in particular. A high index of suspicion is needed to diagnose and properly treat a patient with head and neck infections with concomitant DM.

\section{ACKNOWLEDGEMENTS}

The author acknowledges Dr. Milabelle B. Lingan for providing invaluable contribution, input, encouragement and support in pursuing this research. She served as the clinical consultant to the patients in this study and scientific advisor of this research article.

\section{REFERENCES}

1. Department of Health Philippines. Lifestyle-Related Diseases. [cited 2019 July 21]. Available from: https://www.doh.gov.ph/lifestyle-related-diseases.

2. International Diabetes Federation. The Philippines. [cited 2019 July 21]. Available from: https:// www.idf.org/our-network/regions-members/western-pacific/members/116-the-philippines. html.

3. World Health Organization. Global Report on Diabetes. [cited 2019 July 21]. Available from: https://www.who.int/diabetes/global-report/en/.

4. Sathasivam P. Head and Neck Infections in Diabetic Patients. J Assoc Physicians India. 2018 Sep 66(9):84-88. PubMed PMID: 31321937

5. Delamaire M, Maugendre D, Moreno M, Le Goff MC, Allannic H, Genetet B. Impaired leucocyte functions in diabetic patients. Diabet Med. 1997 Jan; 14(1):29-34. DOI: 10.1002/(SICI)1096136(199701)14:1<29*AID-DIA300>3.0.CO;2-V; PubMed PMID: 9017350

6. Gupta S, Koirala J, Khardori R, Khardori N. Infections in Diabetes Mellitus and Hyperglycemia Infect Dis Clin North Am. 2007 Sep; 21(3):617-38, vii. DOI: 10.1016/j.idc.2007.07.003; PubMed PMID: 17826615.

7. Smit J, Søgaard M, Schønheyder HC, Nielsen H, Frøslev T, Thomsen RW. Diabetes and risk of community-acquired Staphylococcus aureus bacteremia: a population-based case-contro study. Eur J Endocrinol. 2016 May; 174(5):631-9. DOI: 10.1530/EJE-16-0023; PubMed PMID: 26966175

8. Juliana Casqueiro, Janine Casqueiro, and Cresio Alves. Infections in patients with diabetes mellitus: A review of pathogenesis. Indian J Endocrinol Metab. 2012 Mar; 16 Supp 1(Suppl1):S27-36. DOI: 10.4103/2230-8210.94253; PubMed PMID: 22701840; PubMed Central PMCID: PMC3354930

9. Pae M, Meydani SN, Wu D. The role of nutrition in enhancing immunity in aging. Aging Dis. 2012 Feb; 3(1):91-129. PubMed PMID: 22500273; PubMed Central PMCID: PMC3320807.

10. Parhiscar A, Har-El G. Deep neck abscess: a retrospective review of 210 cases. Ann Otol Rhinol Laryngol. 2001 Nov; 110(11):1051-4. DOI: 10.1177/000348940111001111; PubMed PMID: 11713917.

11. Chen MK, Wen YS, Chang CC, Lee HS, Huang MT, Hsiao HC. Deep neck infections in diabetic patients. Am J Otolaryngol. May-Jun 2000; 21(3):169-73. DOI: 10.1016/s0196-0709(00)85019-x PubMed PMID: 10834550.

12. El-Sayed Y, Al Dousary S. Deep-neck space abscesses. J Otolaryngol 1996 Aug;25(4):227-33. PubMed PMID: 8863209.

13. Vieira F, Allen SM, Stocks RMS, Thompson JW. Deep neck infection. Otolaryngol Clin North Am 2008 Jun; 41(3):459-83, vii. DOI: 10.1016/j.otc.2008.01.002; PubMed PMID: 18435993.

14. Sahly $\mathrm{H}$, Podschun $\mathrm{R}$, Ullmann U. Klebsiella infections in the immunocompromised host. Adv Exp Med Biol. 2000; 479:237-49. DOI: 10.1007/0-306-46831-X 21; PubMed PMID: 10897425.

15. Weisenberg SA, Morgan DJ, Espinal-Witter R, Larone DH. Clinical outcomes of patients with Klebsiella pneumoniae carbapenemase-producing K. pneumoniae after treatment with imipenem or meropenem. Diagn Microbiol Infect Dis. 2009 Jun;64(2):233-5. DOI: 10.1016/j. diagmicrobio.2009.02.004. Epub 2009 Apr 2. PubMed PMID: 19345034; PubMed Central PMCID: PMC2764245.

16. Chan YR, Liu JS, Pociask DA, Zheng M, Mietzner TA, Berger T, Mak TW, Clifton MC, Strong RK, Ray P, Kolls JK. Lipocalin 2 is required for pulmonary host defense against Klebsiella infection. J Immunol. 2009 Apr 15;182(8):4947-56. DOI: 10.4049/jimmunol.0803282. PubMed PMID: 19342674; PubMed Central PMCID: PMC2708928.

17. Adams-Haduch JM, Potoski BA, Sidjabat HE, Paterson DL, Doi Y. Activity of temocillin against KPC-producing Klebsiella pneumoniae and Escherichia coli. Antimicrob Agents Chemother 2009 Jun;53(6):2700-1. DOI: 10.1128/AAC.00290-09. Epub 2009 Mar 30. PubMed PMID: 19332667; PubMed Central PMCID: PMC2687206.

18. Gan YH. Host Susceptibility Factors to Bacterial Infections in Type 2 Diabetes. PLoS Pathog 2013; 9(12):e1003794. DOI: 10.1371/journal.ppat.1003794; PubMed PMID: 24385898; PubMed Central PMCID: PMC3873456.

19. Peleg AY, Weerarathna T, McCarthy JS, Davis TM. Common infections in diabetes: Pathogenesis, management and relationship to glycaemic control. Diabetes Metab Res Rev. 2007 Jan; 23(1):3 13. DOI: 10.1002/dmrr.682; PubMed PMID: 16960917

20. Holkom MA, Fu-Qiang X, Alkadasi B, Yang L, Long MX, Mohamed A. Analysis of maxillofacial and neck spaces infection in diabetic and non diabetic patients. Dentist Case Rep, 2018; 2(2):30-6. Corpus ID: 52998664

21. Huang T, Tseng F, Liu TC et al. Deep neck infection in diabetic patients: Comparison of clinical picture and outcomes with nondiabetic patients. Otolaryngol- Head Neck Surg 2005;132:6 DOI:10.1016/j.otohns.2005.01.035 PubMed PMID: 15944569.

22. Leibovici L, Yehezkelli Y, Porter A, Regev A, Krauze I, Harell D. Influence of diabetes mellitus and glycaemic control on the characteristics and outcome of common infections. Diabet Med. 1996 May; 13(5):457-63. DOI: 10.1002/(SICl)1096-9136(199605)13:5<457::AID-DIA83>3.0.CO;2-T PubMed PMID: 8737028 